2. S. N. Mergelyan, On the representation of functions by series of polynomials on closed sets, Doklady Akademii Nauk SSSR. (N.S.) vol. 78 (1951) pp. 405-408; Amer. Math. Soc. Translations, no. 85, Providence, 1953.

3. Walter Rudin, Subalgebras of spaces of continuous functions, Proc. Amer. Math. Soc. vol. 7 (1956) pp. 825-830.

UNIVERSITY OF ROCHESTER

\title{
A SCHWARZ INEQUALITY FOR CONVEX OPERATOR FUNCTIONS
}

\section{CHANDLER DAVIS}

For any Hilbert space $\mathfrak{K}$, let $\mathscr{K}$ be the totality of bounded selfadjoint operators with spectrum contained in an interval $I$, which need not be finite. If $f$ is a function from $\mathfrak{K}$ to the self-adjoint operators on $\mathfrak{F C}$ obtained from a bounded real-valued function $f_{0}$ on $I$ by the spectral theorem (that is, by $f(A)=\int_{-\infty}^{\infty} f_{0}(\lambda) d E_{\lambda}$, where $E_{\lambda}$ is the spectral resolution of $A$ ), then $f$ is called an operator function (associated with $I$ ). An operator function $f$ is defined, once $f_{0}$ is given, for all such $\Re$. An operator function $f$ associated with $I$ is convex provided $A, B$ in $\mathcal{K}$ and $0 \leqq t \leqq 1$ imply

$$
f(t A+(1-t) B) \leqq t f(A)+(1-t) f(B) .
$$

THEOREM. The operator function $f$ associated with $I$ is convex if and only if

$$
\text { (A) for } A \in \mathfrak{K} \text { and for any projection } P, \quad P f(P A P) P \leqq P f(A) P \text {. }
$$

Seymour Sherman suggested to me the problem of characterizing operator functions $f$ satisfying (A) by conditions on the corresponding real-valued functions $f_{0}$. (He remarked that the square is such a function $^{1}$ but the absolute value is not. Sherman has also made an attack on the present theorem by other methods.) The characterization is provided by combining the present theorem with characterizations of convex operator functions [1]. In particular, it is necessary but far from sufficient that $f_{0}$ be convex and analytic.

Proof. From the definition follow these useful facts about any operator function:

Presented to the Society, October 27, 1956; received by the editors March 21, 1956.

$1\left((\mathrm{PAP})^{2} x, x\right)=(P A P x, A P x) \leqq(A P x, A P x)=\left(P A^{2} P x, x\right)$. 
Fact 1. If $P$ is a projection commuting with $A$, then $P f(P A P) P$ $=P f(A) P$.

Fact 2. If $V$ is an isometry, $f\left(V A V^{-1}\right)=V f(A) V^{-1}$.

Another preliminary remark is that the property of convexity is possessed by all the constants (actually by all linear functions), and by the sum of any two functions possessing the property; and correspondingly for property (A). This allows me in the last two paragraphs of the proof to make without loss of generality the convenient assumption $f(0)=0$. For such $f, f(P A P)=P f(P A P) P$; because $P$ commutes with $P A P$, so by Fact $1, \tilde{P} f(P A P) \tilde{P}=\tilde{P} f(0) \tilde{P}=0$. (Here and hereafter, $\tilde{P}$ means $1-P)$. For such $f$, therefore, the inequality in (A) is just $f(P A P) \leqq P f(A) P$.

Assume $f$ a convex operator function, $A \in \mathcal{K}$, and $P$ any projection.

$$
\begin{aligned}
P f(P A P) P= & P f(P A P+\widetilde{P} A \widetilde{P}) P \\
\leqq & \frac{1}{2} P f(P A P+P A \widetilde{P}+\widetilde{P} A P+\widetilde{P} A \widetilde{P}) P \\
& +\frac{1}{2} P f(P A P-P A \widetilde{P}-\tilde{P} A P+\widetilde{P} A \widetilde{P}) P,
\end{aligned}
$$

by Fact 1 and the definition of convexity. But each of the terms on the right is equal to $2^{-1} P f(A) P$-the first trivially, and the second by Fact 2 with $V=P-\tilde{P}$. Hence (A).

Assume (A), $f(0)=0,0 \leqq t \leqq 1$, and $A, B \in \Re$. The proof of (1) will be accomplished in the Hilbert space of pairs

$$
\left(\begin{array}{l}
x_{1} \\
x_{2}
\end{array}\right)
$$

with $x_{1}, x_{2} \in \mathcal{F C}$. This Hilbert space will be denoted $\mathfrak{H C}_{1} \oplus \mathcal{H C}_{2}, \mathfrak{F C}_{1}$ being the subspace of elements having second coordinate zero and $\mathfrak{H}_{2}$ the subspace where first coordinates are zero. Let $V_{\boldsymbol{i}}$ be the natural isometry of $\mathfrak{H C}$ onto $\mathfrak{H C}_{i}$; e.g.,

$$
V_{1}(x)=\left(\begin{array}{l}
x \\
0
\end{array}\right)
$$

It will be convenient to identify $\mathfrak{F}$, not with either $\mathfrak{F}_{1}$ or $\mathfrak{F C}_{2}$, but with a different subspace of $\mathfrak{F C}_{1} \oplus \mathfrak{F C}_{2}$, the projection onto which will be called $P$. Namely, $x \in \mathcal{H C}$ will be identified

$$
x=\left(\begin{array}{c}
\cos \theta x \\
\sin \theta x
\end{array}\right)
$$


where $\theta$ is chosen so $\cos ^{2} \theta=t$. Operators on $\mathfrak{H C}$ are also to be identified with corresponding operators on this subspace into itself, in the natural way.

Now for the proof. Let $x=P x$.

$$
\begin{aligned}
P\left(V_{1} A V_{1}^{-1}+V_{2} B V_{2}^{-1}\right) P x & =\left(\begin{array}{cc}
\cos ^{2} \theta & \cos \theta \sin \theta \\
\cos \theta \sin \theta & \sin ^{2} \theta
\end{array}\right)\left(\begin{array}{ll}
A & 0 \\
0 & B
\end{array}\right)\left(\begin{array}{l}
\cos \theta x \\
\sin \theta x
\end{array}\right) \\
& =\left(\begin{array}{c}
\left(\cos ^{2} \theta A+\sin ^{2} \theta B\right) \cos \theta x \\
\left(\cos ^{2} \theta A+\sin ^{2} \theta B\right) \sin \theta x
\end{array}\right) \\
& =(t A+(1-t) B) x .
\end{aligned}
$$

Also the operator on either side would annihilate any element of $\widetilde{P}\left(\mathcal{H}_{1} \oplus \mathcal{H}_{2}\right)$, by the identification rule. Therefore $P\left(V_{1} A V_{1}^{-1}\right.$ $\left.+V_{2} B V_{2}^{-1}\right) P=t A+(1-t) B$. Similarly $P\left(V_{1} f(A) V_{1}^{-1}+V_{2} f(B) V_{2}^{-1}\right) P$ $=t f(A)+(1-t) f(B)$. But $f\left(P\left(V_{1} A V_{1}^{-1}+V_{2} B V_{2}^{-1}\right) P\right) \leqq P\left(V_{1} f(A) V_{1}^{-1}\right.$ $\left.+V_{2} f(B) V_{2}^{-1}\right) P$, by (A) and Fact 2. Combining the last three expressions gives (1).

COROLlaRY. Let $\mu$ be a completely positive [4] linear mapping from a $C^{*}$-algebra a to operators on a Hilbert space $\mathrm{JC}^{\prime}$, let $f$ be a convex operator function associated with $I, f(0)=0$, and let $A$ be an element of $a$ with spectrum contained in $I$. Then $f(\mu(A)) \leqq \mu(f(A))$.

Proof. There exist a ${ }^{*}$-homomorphism $\rho$ from $a$ to operators on $\mathcal{F}^{\prime}$, and a projection $P$ in $\mathcal{F}^{\prime}$, such that for $B \in Q, \mu(B)=P \rho(B) P[4]$. Then it is easy to justify these steps: $f(\mu(A))=f(P \rho(A) P) \leqq P f(\rho(A)) P$ $=P \rho(f(A)) P=\mu(f(A))$.

\section{BiBLIOGRAPHY}

1. J. Bendat and S. Sherman, Monotone and convex operator functions, Trans. Amer. Math. Soc. vol. 79 (1955) pp. 58-71.

2. R. V. Kadison, A generalized Schwarz inequality and algebraic invariants for operator algebras, Ann. of Math. vol. 56 (1952) pp. 494-503.

3. M. A. Neumark, On a representation of additive operator set functions, C. R. (Doklady) Akad. Sci. URSS. (N.S.) vol. 41 (1943) pp. 359-361.

4. W. F. Stinespring, Positive functions on $C^{*}$-algebras, Proc. Amer. Math. Soc. vol. 6 (1955) pp. 211-216.

5. B. Sz.-Nagy, A moment problem for self-adjoint operators, Acta Mathematica Academiae Scientiarum Hungaricae vol. 3 (1952) pp. 285-293.

KENYON AND ECKHARDT, INC. AND

Columbia University 